\title{
Modeling of the phospholipid vesicular nanostructure formation process
}

\author{
LeSIA AVDIEIEVA ${ }^{1 *}$, OLEKSANDR CHUNIKHIN ${ }^{2}$ \\ ${ }^{1}$ Institute of Engineering Thermophysics, NAS of Ukraine, Kyiv, Ukraine \\ ${ }^{2}$ O.V. Palladin Institute of Biochemistry, NAS of Ukraine, Kyiv, Ukraine
}

\begin{abstract}
This study presents the results of experimental studies and mathematical modeling of the process of vesicular structure formation from phospholipids under the influence of mechanisms of the discrete impulse input of energy (DIIE). The possibility of using this method for increasing the productivity of the process of obtaining vesicles from phospholipids is shown. Moreover, the use of the properties of lipid nanostructures obtained by the DIIE method for the composition of products of special therapeutic nutrition is proposed. The DIIE effect was realized in a flowing rotary-pulsation apparatus of a cylindrical type. The effectiveness of using this type of equipment and the high level of mechanical and physicochemical effects on the dispersed system with phospholipids are established. The results of the studies of the effect of certain regime parameters (such as initial temperature, material concentration, and angular velocity of the rotor) on the analysis of the aqueous suspension of phospholipids on the size distribution of the formed particles are presented. The heat-technological parameters of the process were selected for obtaining particles having an average diameter of up to $500 \mathrm{~nm}$. The possibility of predicting the properties of the formed phospholipids structures obtained by the proposed treatment in a wide range of regime parameters is shown. For the simulation, a hybrid functional Petri net was used, which made it possible to combine the initial thermal technological conditions of the process (such as temperature and material concentration) and the characteristics of the process equipment (such as type of DIIE activator and rotor speed) in the mathematical model.
\end{abstract}

Key words: discrete-pulse input of energy, dispersion, lipid vesicular nanostructures, mathematical modeling, hybrid functional Petri nets

\section{Introduction}

Nanotechnology provides materials with fundamentally new useful properties. These materials provide an opportunity for the active development of various technical areas such as energy, ecology, information technology, engineering, biology, and medicine. At present, all over the world, development of nanotechnology belongs to priority areas and is based on the analysis of the results of significant data sets of theoretical and experimental studies using mathematical modeling. An integrated approach allows to purposefully manage the technological process of the structural formation of nanoma- terials and to ensure rational conditions for its implementation (Tibbals, 2010; Geckeler and Nishide, 2010; Tiwari and Tiwari, 2017; Naito et al., 2018).

In aqueous solutions, under the influence of the energy introduced into the system, phospholipids molecules form closed bilayer structures (vesicles) (Alipour et al., 2017; Yang et al., 2018; Berg et al., 2012). These nanostructures have attracted considerable interest because of their unique properties, namely, the possibility of solubilization of substances of different nature and their direct transportation into biological cells. The vesicle shell protects the active substance from the influ-

\footnotetext{
${ }^{*}$ Corresponding author: Institute of Engineering Thermophysics, NAS of Ukraine, Kyiv, Ukraine; e-mail: avdeeva22@ukr.net
} 
ence of negative external factors, has an affinity for cell membranes, and is biodegradable; therefore, it significantly increases the positive effect of the use of this form of nanopreparations (McClements and Gumus, 2016; Vance and Vance, 2008; Eliaz, 2011; Berg et al., 2012; Vitkova and Petrov, 2013).

Currently, vesicular structures are obtained from phospholipids using methods such as ultrasonic processing, filtering, injection methods and high-pressure homogenization (Tibbals, 2010). These methods enable obtaining nanostructures with predetermined properties; however, they have several disadvantages such as low productivity and considerable energy consumption (Avdieieva, 2011). Consequently, using phospholipid nanostructures is limited to basic research as a model of cell membranes, as well as the production of drugs for diagnosis and treatment in medical and pharmaceutical industries (McClements and Gumus, 2016; Nii and Ishii, 2005; Eliaz, 2011; Vitkova and Petrov, 2013). Furthermore, the use of phospholipids vesicles in the food industry and agriculture to obtain nanopreparations with increased efficiency seems very promising (Mozafari et al., 2006, Sanguansri, 2006; Cushen et al., 2012; Tsai, 2016). The increased interest in the potential of phospholipid vesicles and the expansion of their range of applications requires the presence of highly productive equipment and development of energy-efficient industrial technology for producing new products (Sanguansri and Augustin, 2006; Avdieieva, 2011; Cushen et al., 2012; Berekaa, 2015; Tsai, 2016).

The solution to the problem of reducing energy intensity and increasing the productivity of processes in developing industrial technology for producing lipid nanostructures is achieved using equipment that implements the mechanisms of discrete impulse input of energy (DIIE). The DIIE principle was proposed in the early 1980 s as a generalizing method of directional, local, and intensive use of concentrated energy in compressible vapor-liquid heterogeneous disperse systems. Subsequently, there has been considerable research on its successful use in various industries (Dolinskyi, 2008).

The DIIE principle determines the paths of the conversion of the continuously and randomly distributed energy introduced in the apparatus into short-duration high-power pulses, which are discretely distributed in the working volume of the system being processed. During the practical implementation of the DIIE, the phenomena that occur in the working volume of a dispersed system include explosive boiling up, as well as hydrodynamic, acoustic, and steam cavitation. Moreover, DIIE initiates the targeted use of powerful dynamic effects with accompanying high-frequency oscillations, spherical shock waves, and cumulative microstructures. Thus, a high level of intensification of the mass transfer and hydromechanical processes is achieved with a minimal waste of energy, thus maintaining high quality material processing (Alipour et al., 2017; Yang et al., 2018; Nii and Ishii, 2005; Sanguansri and Augustin, 2006; Cushen et al., 2012; Tsai, 2016). To date, a number of devices operating on this principle (such as emulsifiers, rotary pulsation apparatus, pulsators and cavitators) and a large number of technologies allowing to intensify various technological processes have been developed (Dolinskyi, 2008). Such devices are highly efficient that allow to obtain a multifactorial pulse effect on such types of dispersed systems as "liquid-liquid", "liquid-solid", or "gas-liquid." The DIIE principle has been successfully used for the processes of mixing, crushing, emulsifying, and homogenizing to significantly increase the total contact surface of the components and increase the homogeneity and stability of the disperse system (Dolinskyi, 2008).

While obtaining phospholipid vesicles, the process of structure formation and the properties of the formed vesicles was controlled by thermodynamic and hydrodynamic parameters of the process, i.e., pressure, temperature, flow rate, angular velocity of the rotor, duration of treatment, as well as the composition of phospholipids (Nii and Ishii, 2005; Berg et al., 2012; Tsai, 2016). Therefore, the properties of the particles obtained depend largely on the characteristics of the equipment (such as power, angular velocity, and volume), technological parameters of processing of the dispersed system (such as pressure, concentration, and temperature), as well as the qualitative and quantitative composition of phospholipids.

In this study, we aim to examine, using the DIIE method, the effects of certain operating parameters of the processing of phospholipids aqueous suspensions (such as initial temperature, material concentration, and rotor angular velocity) on the properties of formed particles that can be used in industrial technology for producing medicinal products. 


\section{Materials and methods}

\section{Materials}

Dry soy lecithin Solaye (Solaye, EU) with a total phospholipids content of $97 \%$ was used. This phospholipid preparation has been approved for use in the food industry, has the properties to form persistent microemulsions in oil-in-water systems, and has been characterized by a high total content of phosphatidylcholine (35.4\%) and phosphatidyl ethanolamine (21.8\%), both of which are required for forming a closed vesicle membrane (Vance and Vance, 2008; Dyńska-Kukulska and Ciesielski, 2012).

\section{Methods}

1. A flow-type rotary-pulsation apparatus of cylindrical type, which consists of two stators and a rotor with 24 slit-shaped holes, developed at the Institute of Technical Thermophysics of the National Academy of Sciences of Ukraine was used for this study (Dolinskyi, 2008; Avdieieva, 2011). The nominal value of the angular velocity of the rotor is $314 \mathrm{rad} / \mathrm{s}$. The processing was carried out with three cycles of passage of the material through the working bodies of the apparatus.

2. The particle size distribution function was investigated using dynamic light scattering on a laser photon-correlation spectrometer “ZetaSizer-3" Malvern Instruments, UK (Merkus, 2009; McNeil, 2011). The recording and statistical processing of the changes in the scattering intensity in the particle water suspension $(n=1.33)$ were performed ten times for $10 \mathrm{~min}$ at $+22^{\circ} \mathrm{C}$ at a scattering angle of $90^{\circ} \mathrm{C}$. The obtained results were processed using the PCS-Size mode v1.61 software using the CONTIN algorithm.

3. Under the conditions of a multifactor experiment, it is difficult to obtain a result over the entire range of variations in the parameters of the process. Depending on the conditions of their formation in the whole range of variability of the parameters of the technological process, the determination of quantitative indicators of lipid vesicles (size and internal volume), was performed using mathematical modeling (Nagasaki et al., 2007; Koch et al., 2011). This approach allowed us to consider a large number of physical factors and provide a prediction of the behavior of a dispersed system in a wide range of operating parameters.
The Cell Illustrator v.3 software environment (Human Genome Center, University of Tokyo, Japan) was selected as a tool for modeling the mechanism of formation of vesicular phospholipid nanostructures, in which hybrid functional Petri nets are the basic modeling tool (Nagasaki et al., 2007; Koch et al. 2011). The advantages of hybrid functional Petri nets as modeling tools are as follows:

- the possibility of a structural display of the state of the system that is modeled, along with the processes that occur in the system;

- the possibility of quantitative modeling of states and processes of three types at the same time: discrete, continuous, and associative (generatrix);

- the possibility of considering the activating, inhibiting, and catalytic effects using special type of bonds. Petri nets are a mathematical abstraction for representing discrete and continuous distributed systems graphically represented as a bipartite oriented multigraph with markers (marked directed graph), which has two types of vertices: positions $\mathrm{P}$ and transitions T. Positions can be either empty or marked and they determine the state of the network while transitions show the action (process).

The operation of the Petri net depends on the sequential execution of transitions and the corresponding recalculation of the number of "resource" in the positions (Fig. 1)

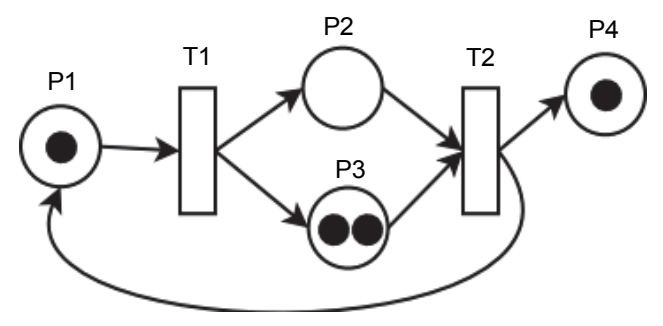

Fig. 1. Schematic of the Petri net

The efficiency of inclusion (Xls) is one of the most important indicators of lipid vesicular preparations, which characterizes the amount of the substance in the middle of the vesicle. This indicator can be calculated using the following formula (Merkus, 2009):

$$
X_{l s}=\frac{C \cdot(D-h)^{3} \cdot N_{A} \cdot S \cdot 10^{-27}}{\left(D^{2}+(D-h)^{2}\right) \cdot M r \cdot 6}
$$


where $C$ is the concentration of phospholipids, $\mathrm{mg} / \mathrm{ml}$; $D$ is the diameter of the vesicle, $\mathrm{A} ; h$ is the monolayer thickness, A; $N_{A}$ is the Avogadro number, and $S$ is the area of the phospholipid molecule in the monolayer, $\mathrm{A}^{2}$; and $M r$ is the molar mass of the phospholipid.

\section{Results}

Size is the most important property of lipid vesicles (Cevc, 1993; Vance and Vance, 2008; Tsai, 2016; Vitkova and Petrov, 2013). Our task was to obtain, because of the proposed treatment, primarily large single-layer particles with an average size of $400-500 \mathrm{~nm}$, all of which have a large internal volume and are characterized by persistence during the entire shelf life of the food product.

Figure 2 shows the results of the studies of samples with a concentration of lecithin in the range from $0.5 \%$ to $7.5 \%$ obtained at a temperature of $42 \pm 2{ }^{\circ} \mathrm{C}$.

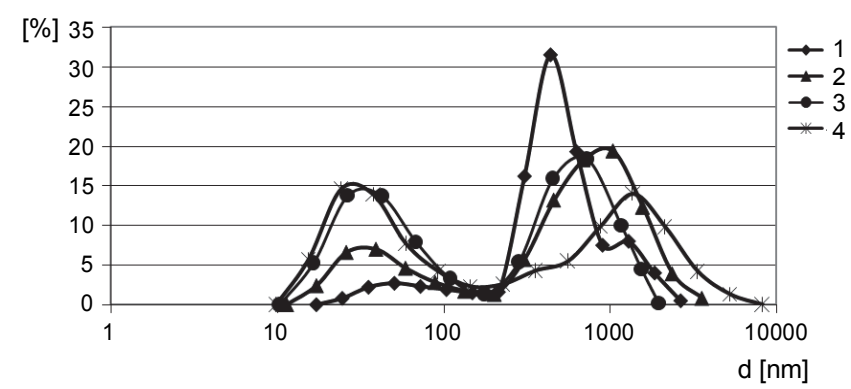

Fig. 2. Distribution of particle sizes (diameter) of the tested samples obtained at temperature of $42 \pm 2{ }^{\circ} \mathrm{C}$ with concentrations of phospholipids: sample $1-0.5 \%$; sample $2-2.5 \%$; sample $3-5 \%$; sample $4-7.5 \%$

Experimental results showed that in all the samples that were obtained and tested, the particles had a bimodal distribution of diameter from $10-15 \mathrm{~nm}$ to $450-500 \mathrm{~nm}$ and from 300 to $2.0 \mathrm{~nm}$ and above. These results may indicate the absence of micelles and the presence of large single-layer and multi-layer vesicles (Evans and Skalak, 1980; Vance and Vance, 2008; Naito et al., 2018). In a sample with a $0.5 \%$ concentration of phospholipids, large particles were predominant, $82.5 \%$ of which had sizes ranging from 300 to $3.0 \mu \mathrm{m}$. Increasing the concentration of phospholipids to $2.5 \%$ led to a decrease in the overall dispersion range with small and large single-layer vesicles with sizes of up to $1000 \mathrm{~nm}$ forming $83 \%$ of the sample size. In a sample with a $5 \%$ concentration of phospholipids, the dispersion range of the particles was further narrowed and large single-layer vesicles with sizes of up to $1000 \mathrm{~nm}$ formed more than $86 \%$ of the sample size. The average particle diameter of this sample was $440 \mathrm{~nm}$, and the studies of samples with a phospholipid concentration of $7.5 \%$ showed an increase in the number of small single-layer vesicles with sizes up to $100 \mathrm{~nm}$ to $46 \%$; however, there was a significant expansion of the dispersion range up to $3.0 \mu \mathrm{m}$. This may indicate deterioration of the conditions of dispersion and significant aggregation of particles (Evans and Skalak, 1980; Vance and Vance, 2008). Thus, the most rational possibility was to use a $5 \%$ concentration of phospholipids in the dispersed system.

Next, we studied the effect of temperature processing parameters on the size of the particles formed. Figure 3 shows the results of experimental studies of samples with $5 \%$ concentration of phospholipids obtained at dispersion temperatures ranging from 20 to $80^{\circ} \mathrm{C}$.

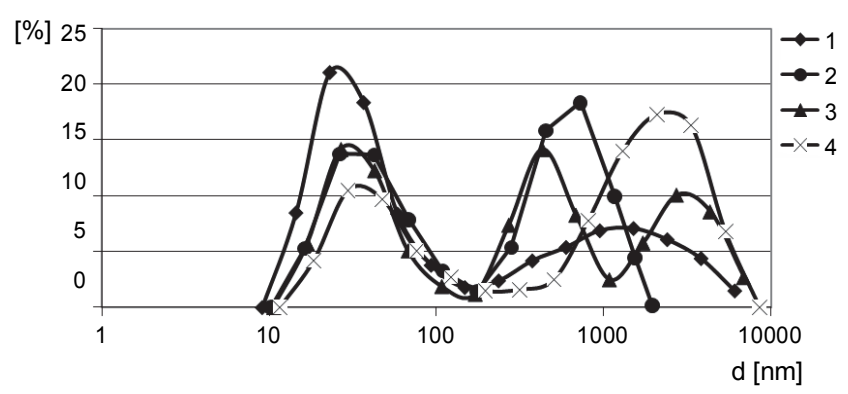

Fig. 3. Particle size distribution of samples obtained at a phospholipid concentration of $5 \%$ and a temperature of dispersion in ranges: sample $1-20^{\circ} \mathrm{C}$; sample $2-42^{\circ} \mathrm{C}$; sample $3-60^{\circ} \mathrm{C}$; sample $4-80^{\circ} \mathrm{C}$

The results showed (Fig. 3) that the sizes of the particles formed had a bimodal distribution with a significant number of particles in the range of up to $100 \mathrm{~nm}$. However, almost all samples were characterized by significant dispersion. For a sample obtained at $20^{\circ} \mathrm{C}(1)$, this was probably because of the peculiarities of the behavior of phospholipid suspensions at low temperatures. Increasing the temperature to $42 \pm 2^{\circ} \mathrm{C}$ (sample 2), which corresponded to the phase transition temperature for most phospholipids, translated the dispersed system into a liquid-crystalline state and weakened the intermolecular bonds (Cevc, 1993; Hanahan, 1997; Vance and Vance, 2008). This approach allowed to improve the condition of the dispersion and to obtain smaller particle sizes. The sample obtained as a result of treatment at 
$60^{\circ} \mathrm{C}$ (sample 3 ) was characterized by a three-modal distribution of particles and an increase in the range of dispersion to $7.0 \mu \mathrm{m}$, which may indicate the aggregation of particles after dispersion. Note that sample 4 had a bimodal particle distribution with $54.4 \%$ of particles having sizes larger than $1 \mu \mathrm{m}$. Thus, for further experimentation, the temperature dispersion range of $42 \pm 2^{\circ} \mathrm{C}$ was used.

By using a belt transmission, the angular velocity of the DIIE activator was successively increased from 314 to 472 and $628 \mathrm{rad} / \mathrm{s}$. Figure 4 shows the results of experimental studies of the effect of the angular velocity of the rotor in the course of DIIE-processing on the size of the formation of particles. During production, the samples with a phospholipid concentration of $5 \%$, obtained at dispersion temperatures of $42 \pm 2^{\circ} \mathrm{C}$, were used.

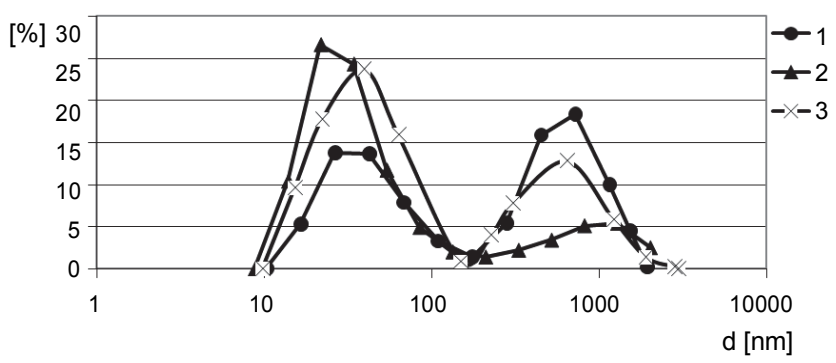

Fig. 4. Particle size distribution of the obtained samples with a concentration of $5 \%$ at a dispersion temperature of $42 \pm 2{ }^{\circ} \mathrm{C}$ with an angular speed of the rotor: sample $1-314 \mathrm{rad} / \mathrm{s}$, sample 2 - $472 \mathrm{rad} / \mathrm{s}$, sample $3-628 \mathrm{rad} / \mathrm{s}$

The results show an increase in the impact on the material being processed with an increase in the angular velocity of rotation of the rotor for this type of DIIEactivator. Therefore, at a speed equal to $314 \mathrm{rad} / \mathrm{s}$, particles with a size of up to $500 \mathrm{~nm}$ formed $70 \%$ of the dispersion of the entire system, i.e., an increase in speed to $472 \mathrm{rad} / \mathrm{s}$ led to an increase in the percentage of such particles to $86.7 \%$. A further increase in speed up to $628 \mathrm{rad} / \mathrm{s}$ led to the simultaneous sticking of small particles into conglomerates as well as the grinding of large particles. Consequently, the number of particles with a size of up to $500 \mathrm{~nm}$ slightly decreased and accounted for $80 \%$ of the dispersity of the entire system. The results show that increasing the angular velocity of the rotor to $472 \mathrm{rad} / \mathrm{s}$ and $628 \mathrm{rad} / \mathrm{s}$ led to an improvement in the dispersion conditions and a larger number of particles in the specified range up to $500 \mathrm{~nm}$ were obtained. However, these conditions led to a significant increase in energy consumption for material processing, as well as required additional strengthening of certain parts to preserve the durability and operability of the equipment.

To analyze the obtained results and to formulate general laws of the influence of the thermal parameters on the change in the size of vesicular nanostructures during the process of DIIE processing, the expected value of the particle size was calculated. Figure 5 shows the results of the calculation of the dependence of the average particle diameter on the concentration of phospholipids at different temperatures of dispersion with an angular velocity of $314 \mathrm{rad} / \mathrm{s}$.

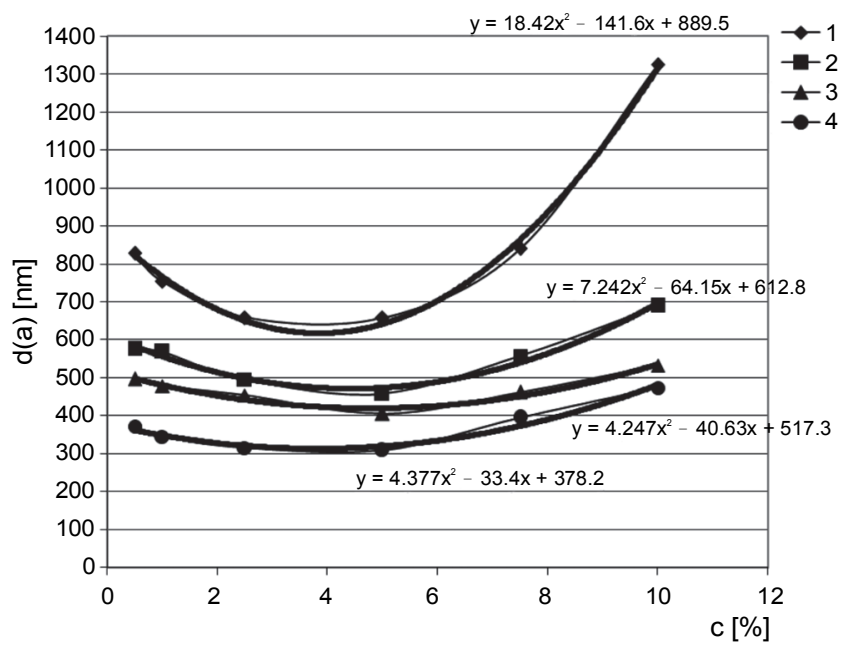

Fig. 5. The dependence of the average particle diameter, obtained with an angular velocity of $314 \mathrm{rad} / \mathrm{s}$, on the concentration of phospholipids at the following temperatures of DIIE treatment: sample $1-20^{\circ} \mathrm{C}$; sample $2-42^{\circ} \mathrm{C}$; sample $3-60^{\circ} \mathrm{C}$; sample $4-80^{\circ} \mathrm{C}$

Figure 5 shows the results of the analysis of the measurements presented, and they show a significant effect of the thermal parameters during DIIE processing on the value of the average diameter of the particles formed. In particular, this dependence is traced to curve 1 , at $20^{\circ} \mathrm{C}$, where because of a change in the concentration of the material from $0.5 \%$ to $5 \%$, the average particle size gradually decreased 1.25 times from 827 to $657 \mathrm{~nm}$. An increase in the concentration of the material to $>5 \%$ led to a gradual increase in the average particle diameter from 827 to $1324 \mathrm{~nm}$ (1,6 times), possibly because of the behavior of phospholipids at temperatures below the phase transition temperature. In samples 2,3 , and 4 , 
which were obtained at higher temperatures of the homogenization process, a change in the concentration of the material led to less significant changes in the average particle size, but the general tendencies remained. The lowest diameter was observed after dispersing the lipid material at $5 \%$ concentration. A further increase in concentration (by 2 times) from 5 to $10 \%$ led to a gradual increase in the diameter of 1.3-1.5 times.

An increase in the homogenization temperature to $42^{\circ} \mathrm{C}$ (sample 2) led to the transfer of the dispersion with phospholipids from the solid crystalline state to the liquid crystal state and weakening of the intermolecular bonds. This in turn led to a decrease in the average diameter of the particles formed (Cevc, 1993; Hanahan, 1997; Vance and Vance, 2008). A further increase in the temperature of the process led to a further weakening of the bonds between the molecules of the lipid material; therefore, the average diameter of the particles obtained decreased, and the efficiency of homogenization improved. Thus, for all samples tested, increasing the temperature of the process to $60^{\circ} \mathrm{C}$ (sample 3 ) and $80^{\circ} \mathrm{C}$ (sample 4) led to an increase in its efficiency and a decrease in the dispersion of the system.

Thus, the analysis of the results obtained indicates and confirms the possibility of controlling the process of the formation of vesicles from phospholipids under the conditions of application of the DIIE mechanisms using rational heat-engineering regime parameters (Avdieieva, 2011).

\section{Mathematical modeling}

Modeling the mechanism of formation and self-organization of vesicular phospholipid nanostructures under the DIIE influence was aimed at systematically integrating in the mathematical model both the initial technological parameters of the material (such as temperature and concentration) and the characteristics of the equipment used (such as DIIE-activator and rotation frequency rotor). Based on the results of experimental studies of the average diameter of lipid vesicles on the conditions and modes of the technological process of their production, partial functional dependencies were observed. As approximating functions, polynomials from the second to the fifth degree were used.

The approximating dependences of the average diameter (Dav) of lipid vesicles on the temperature $(i)$ and the concentration of phospholipids $(c)$ at different an- gular speeds of rotation of the rotor $(\omega)$ are shown in the equations $2-4$ :

$$
\begin{aligned}
& \text { at } \omega=3,000 \mathrm{rpm}=314 \mathrm{rad} / \mathrm{s} \\
& \begin{aligned}
D_{a v} & =\left(67 \times 10^{-4} \times t^{2}-0.8951 t+33.536\right) c^{2}- \\
& +\left(0.041 t^{2}-5.865 t+241.59\right) \times c-8.2877 t+1018
\end{aligned}
\end{aligned}
$$

at $\omega=4,500 \mathrm{rpm}=472 \mathrm{rad} / \mathrm{s}$ :

$$
\begin{aligned}
D_{a v} & =\left(4 \times 10^{-5} \times t^{3}-53 \times 10^{-4} t^{2}-0.2074 t+5.5137\right) c^{2}- \\
& +\left(5 \times 10^{-4} t^{3}-0.0729 t^{2}+3.108 t+49.963\right) c+ \\
& +\left(18 \times 10^{-4} t-0.2547 t^{2}+9.4619 t+392.96\right)
\end{aligned}
$$$$
\text { at } \omega=6,000 \mathrm{rpm}=628 \mathrm{rad} / \mathrm{s} \text { : }
$$

$D_{a v}=\left(4 \times 10^{-5} \times t^{3}-84 \times 10^{-4} t^{2}-0.5627 t-6.8177\right) c^{2}-$

$+\left(6 \times 10^{-4} t^{3}-0.1034 t^{2}+6.7957 t-81.854\right) c+$

$+\left(3 \times 10^{-4} t-0.0571 t^{2}+3.4706 t+349.81\right)$

The results of the studies of partial functional dependences of the average diameter of lipid vesicles on the parameters of the technological process made it possible to parameterize the Petri net. Moreover, using Petri net, a graph-analytical model was built, which allows calculating the average diameter of lipid nanostructures obtained because of the processing of a lipid suspension depending on the rotor speed of the DIIE-activator, and the concentration and the temperature of the starting material (Fig. 6). Thus, equations 2-4 parameterized the p1-p3 transitions, respectively, which made it possible to obtain the numerical value of the vesicles average diameter "GDDave" at the $\mathrm{m} 5$ position.

Table 1 shows the examples of calculating the diameter of phospholipid vesicles according to the results of studies obtained experimentally (De) and analytically $(\mathrm{Dm})$, using Petri nets, at a temperature $t=42 \pm 2^{\circ} \mathrm{C}$, material concentration $(c)$ from 0.5 to $10 \%$ and rotor angular speeds $(\omega)$ of 314,472 , or $628 \mathrm{rad} / \mathrm{s}$. The absolute error of the simulation was calculated using the formula: $\Delta=|\mathrm{Dm}-\mathrm{De}|, \mathrm{nm}$, and the relative modeling error was calculated using the formula: $\delta=(\Delta / \mathrm{De}) \times 100 \%$.

Thus, the proposed analytical model developed on the basis of the simulation environment "Hybrid Functional Petri Nets" makes it possible to accurately calculate the value of the average diameter of lipid vesicles depending on a wide range of processing parameters: temperature (from 20 to $80^{\circ} \mathrm{C}$ ), material concentration (from 0.5 to $10 \%$ ), with the angular speed at 314,472 , or $628 \mathrm{rad} / \mathrm{s}$. The average relative error of modeling in all modes and conditions was $4.3 \%$. 


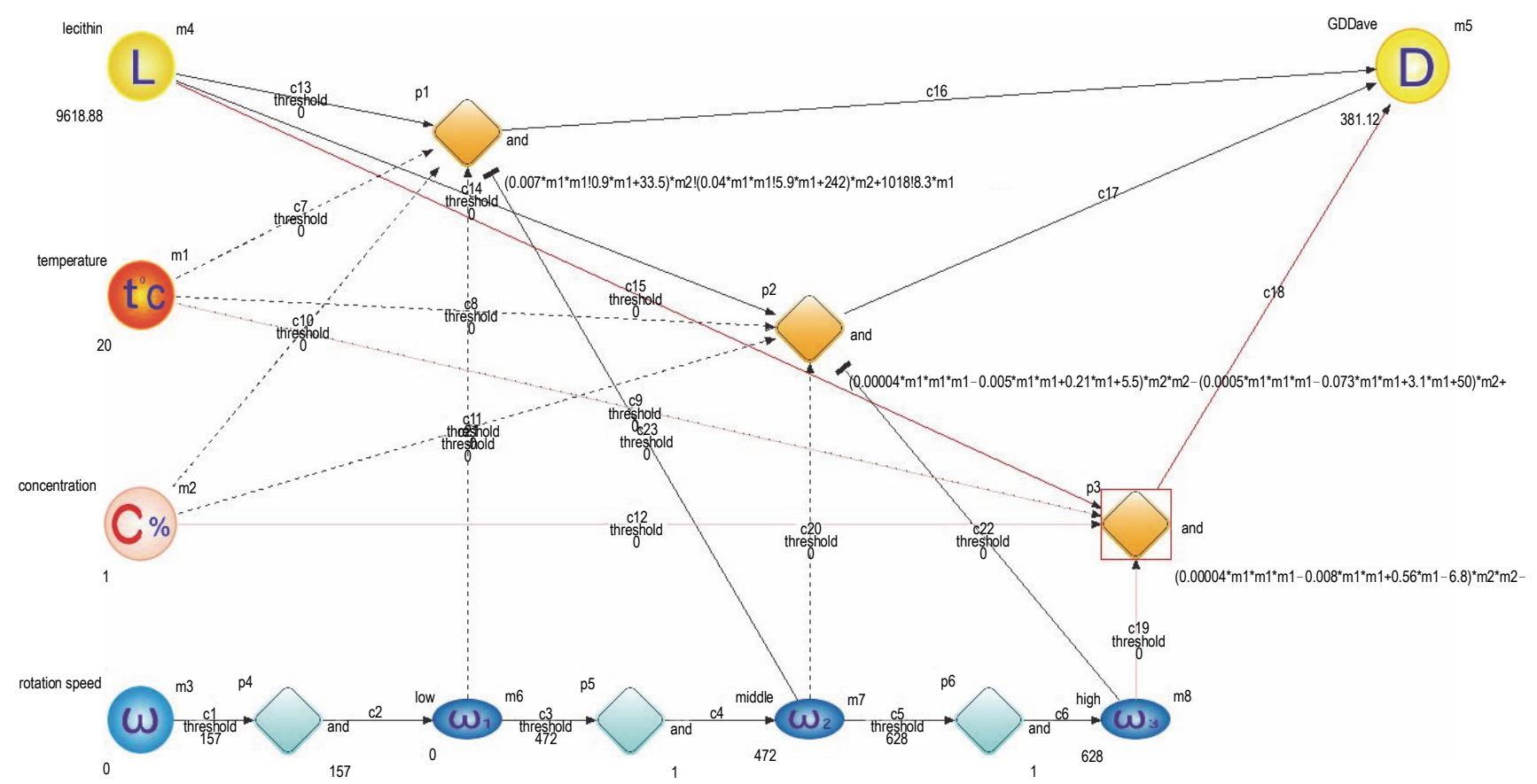

Fig. 6. Graph-analytical model of calculation of the average diameter of lipid vesicles with Petri nets at the concentration of 1\%, temperature of $20^{\circ} \mathrm{C}$, speed of rotor of $628 \mathrm{rad} / \mathrm{s}$

Table 1. The diameter of the phospholipid vesicles obtained experimentally and analytically using Petri nets

\begin{tabular}{|c|c|c|c|c|c|c|}
\hline $\begin{array}{c}\text { Temperature } \\
{\left[{ }^{\circ} \mathrm{C}\right]}\end{array}$ & $\begin{array}{c}\text { Rotor speed } \\
{[\mathrm{rad} / \mathrm{s}]}\end{array}$ & $\begin{array}{c}\text { Concentration } \\
{[\%]}\end{array}$ & $\begin{array}{c}\text { Model } \\
\text { diameter } \\
{[\mathrm{nm}]}\end{array}$ & $\begin{array}{l}\text { Experimental } \\
\text { diameter } \\
{[\mathrm{nm}]}\end{array}$ & $\begin{array}{l}\text { Absolute } \\
\text { error } \\
\text { [nm] }\end{array}$ & $\begin{array}{c}\text { Relative } \\
\text { error } \\
{[\%]}\end{array}$ \\
\hline \multirow{12}{*}{42} & \multirow{4}{*}{314} & 0.5 & 603 & 575 & 28 & 4.87 \\
\hline & & 2.5 & 517 & 496 & 21 & 4.23 \\
\hline & & 5 & 427 & 441 & 14 & 3.17 \\
\hline & & 10 & 722 & 689 & 33 & 4.79 \\
\hline & \multirow{4}{*}{472} & 0.5 & 456 & 441 & 15 & 3.40 \\
\hline & & 2.5 & 301 & 302 & 1 & 0.33 \\
\hline & & 5 & 226 & 225 & 1 & 0.44 \\
\hline & & 10 & 369 & 350 & 19 & 5.43 \\
\hline & \multirow{4}{*}{628} & 0.5 & 386 & 380 & 6 & 1.58 \\
\hline & & 2.5 & 299 & 314 & 15 & 4.78 \\
\hline & & 5 & 234 & 245 & 11 & 4.49 \\
\hline & & 10 & 312 & 330 & 18 & 5.45 \\
\hline
\end{tabular}

\section{Efficiency of inclusion estimation}

Setting the initial data for lipid vesicles: the monolayer thickness $h=40$ and $\mathrm{A}=4 \mathrm{~nm}$, and the area of the phospholipid molecule in the monolayer was $S=60 \mathrm{~A}^{2}$, i.e., the molar mass of the phospholipid $M r=1000 \mathrm{~g} / \mathrm{mol}$ and lipid concentration (\%) after simple transformations.
We then an obtained an expression for the dependence of the inclusion efficiency on the diameter of the vesicles in nanometers $\left(\mathrm{D}_{\mathrm{nm}}\right)$ and lipid concentrations $(\mathrm{C} \%)$ :

$$
X_{l s}=\frac{6 \cdot 10^{-4} \cdot\left(D_{n m}-4\right)^{3}}{D_{n m}^{2}+\left(D_{n m}-4\right)^{2}}
$$




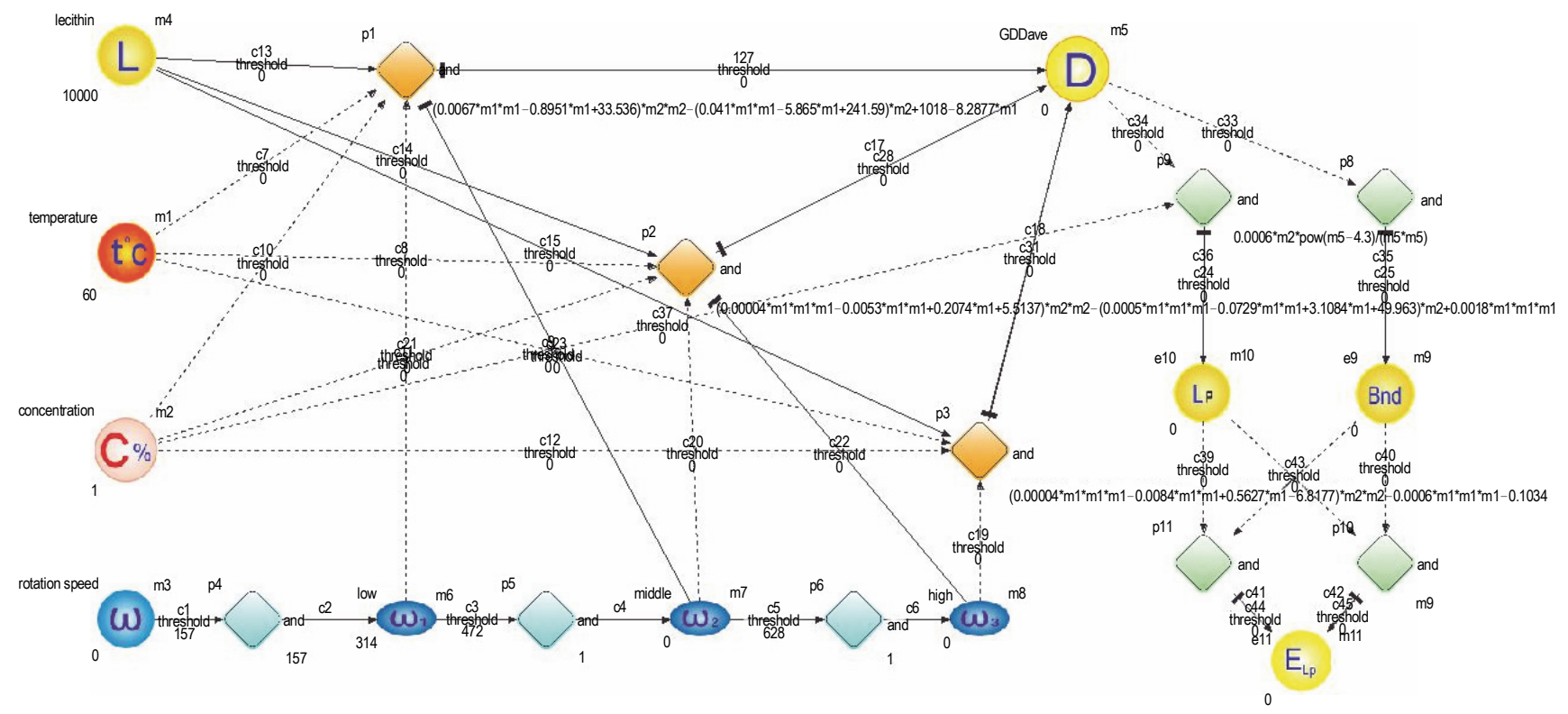

Fig. 7. The calculation of the vesicles average diameter and the effective integration of polar substances using Petri net at the concentration of $1 \%$, temperature of $20^{\circ} \mathrm{C}$, speed of rotor of $628 \mathrm{rad} / \mathrm{s}$

However, there is a theoretical limit to the effectiveness of the inclusion, which is determined by the ratio of the internal volume of the vesicles and its total volume for a given diameter and thickness of the lipid bilayer. Considering the lipid vesicle as an ideal ball with a diameter $D$ and an internal cavity with a diameter $(D-2 \mathrm{~h})$, it is easy to determine the ultimate inclusion efficiency $X_{\dot{t}}$ :

$$
X_{t}=\left(1-\frac{2 h}{D}\right)^{3}
$$

Note that the limits of the application of dependences (1) and (5) are completely determined by expression (6). Therefore, for vesicles with a diameter of $>.200 \mathrm{~nm}$ for medium and high concentrations of phospholipids, it is necessary to compare the calculated values of the efficiency of inclusion with the theoretical limit. Moreover, when considering single-layer vesicles, it is advisable to limit the diameter to $600 \mathrm{~nm}$.

The developed mathematical model was supplemented by dependences that allow us to calculate the inclusion efficiency indicator. For this purpose, the corresponding positions and transitions were added to the existing structure (Fig. 7).

Thus, dependences (5) and (6) parameterized the p9 and $\mathrm{p} 8$ transitions, respectively, which makes it possible to obtain the calculated values of the inclusion efficiency at position $\mathrm{m} 10$ ("Lp") and the marginal efficiency at inclusion at position $\mathrm{m} 9$ ("Bnd"). The final value of the efficiency of inclusion of substances in the vesicles in the position $\mathrm{m} 11$ ("ELp") was defined as the minimum value between the calculated and limiting values of the effectiveness of the inclusion. The automatic selection of the minimum value was ensured by the corresponding thresholds established between positions $\mathrm{m} 9$ and $\mathrm{m} 10$ and transitions $\mathrm{p} 10$ and $\mathrm{p} 11$.

The work of the Petri net comprises two stages. The first one calculates the values of the average diameter of the "GDDave" vesicles depending on the angular velocity of the rotation of the DIIE-activator, the temperature, and the concentration of the starting material. In the second, on the basis of the average diameter of the vesicles and the concentration of lipids, the values of the effectiveness of the inclusion of substances in the vesicles ("ELP") are calculated.

The obtained results of calculation of the vesicles average diameter and the effective integration of polar substances using the Petri net (concentration of $1 \%$, temperature of $20^{\circ} \mathrm{C}$, speed of rotor of $628 \mathrm{rad} / \mathrm{s}$ ) agree well with the results of experimental studies (Fig. 5).

\section{Conclusions}

The possibility of controlling the process of the formation of vesicles from phospholipids under the conditions of the dissipative state of the heterogeneous system using DIIE mechanisms was investigated and pre- 
sented. Depending on the heat-engineering parameters and modes for DIIE treatment, the study and analysis of changes in the properties of nanostructures made it possible to choose rational indicators for obtaining lipid vesicles with certain physical properties.

The mathematical model created using hybrid functional Petri nets made it possible to determine the peculiarities of the formation of vesicular phospholipid nanostructures under the influence of DIIE effects and to obtain the value of the average diameter of vesicles and the efficiency of incorporation in a wide range of process parameters without additional costs for obtaining vesicles and experimental measurement of their size using complex technical means. The advantage of the proposed approach is the possibility of obtaining values of the average diameter of lipid vesicles both in the "nodes" of tabulated values and in the whole range of variability of the process parameters.

\section{References}

Alipour E., Halverson D., McWhirter S. et al. (2017) Phospholipid bilayers: stability and encapsulation of nanoparticles. Ann. Rev. Phys. Chem. 68: 261-283.

Avdieieva L.I. (2011) Energy-efficient technology for the production of phospholipid nanostructures. Industr. Heat Eng. 33(8): 139-143 (in Ukrainian).

Berg J.M., Tymoczko J.L., Stryer L. (2012) Biochemistry, 5th ed. New York: W.H. Freeman.

Cushen M., Kerry J., Morris M., Cruz-Romero M., Cummins E. (2012) Nanotechnologies in the food industry-Recent developments, risks and regulation. Trends Food Sci. Technol. 24(1): 30-46.

Dolinskyi A.A. (2008) Heat-mass transfer and hydrodynamics in steam-liquid dispersive environments. Kiev: Sci. Opin. (in Russian).

Dyńska-Kukulska K., Ciesielski W. (2012) Methods of extraction and thin-layer chromatography determination of phospholipids. Rev. Anal. Chem. 31(1): 43-56.
Eliaz N. (2011) Applications of electrochemistry and nanotechnology in biology and medicine I. New York: Springer Science + Business Media, LLC.

Geckeler K.E., Nishide H. (2010) Advanced nanomaterials. Weinheim: Wiley-VCH Verlag GmbH \& Co. KGaA.

Koch I., Reisig W., Schreiber F. (eds.) (2011) Modeling in systems biology. The Petri net approach. Springer.

McClements D., Gumus C. (2016) Natural emulsifiers - biosurfactants, phospholipids, biopolymers, and colloidal particles: molecular and physicochemical basis of functional performance. Adv. Colloid Interface Sci. 234: 3-26.

McNeil S.E. (2011) Characterization of nanoparticles intended for drug delivery. Humana Press.

Merkus H.G. (2009) Particle size measurements. Fundamentals, practice, quality. Springer.

Nagasaki M. et al. (2007) Foundations of systems biology. Using cell illustrator and pathway databases. Springer.

Naito M., Yokoyama T., Hosokawa K., Nogi K. (2018) Nanoparticle technology handbook, third edition. Elsevier.

Nii T., Ishii F. (2005) Encapsulation efficiency of water-soluble and insoluble drugs in liposomes prepared by the microencapsulation vesicle method. Intern. J. Pharmaceut. 298(1): 198-205.

Sanguansri P., Augustin M.A. (2006) Nanoscale materials development - a food industry perspective. Trends Food Sci. Technol. 17(10): 547-556.

Tibbals H.F. (2010) Medical nanotechnology and nanomedicine. CRC Press.

Tiwari A., Tiwari A. (2017) Bioengineered nanomaterials. CRC Press Published.

Tsai W. (2016) Liposomal microencapsulation using the conventional methods and novel supercritical fluid processes, Trends Food Sci. Technol. 55: 61-71.

Vance J.E., Vance D. (2008) Biochemistry of lipids, lipoproteins and membranes, 5 th ed.

Vitkova V., Petrov A.G. (2013) Lipid bilayers and membranes: material properties. [in:] Advances in planar lipid bilayers and liposomes, vol. 17, Academic Press, Burlington: 89-138.

Yang Y., Lee M. and Fairn G. (2018) Phospholipid subcellular localization and dynamics. J. Biol. Chem. 293(17): 6230-6240. 